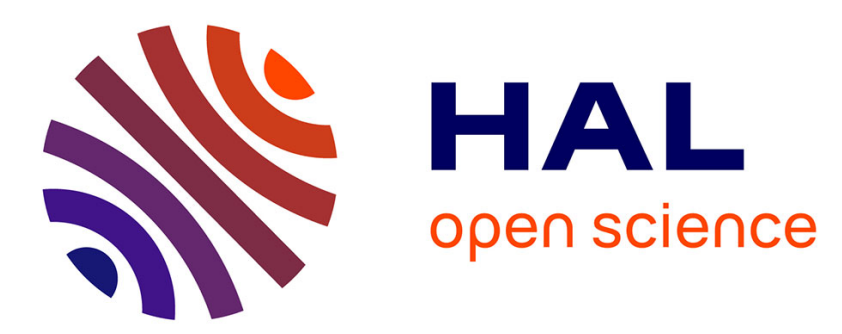

\title{
Biodiversity knowledge synthesis at the European scale: actors and steps
}

\author{
Barbara Livoreil, Ilse Geijzendorffer, Andrew S. Pullin, Stefan Schindler, \\ Marie Vandewalle, Carsten Nesshoever
}

\section{- To cite this version:}

Barbara Livoreil, Ilse Geijzendorffer, Andrew S. Pullin, Stefan Schindler, Marie Vandewalle, et al.. Biodiversity knowledge synthesis at the European scale: actors and steps. Biodiversity and Conservation, 2016, 25 (7, SI), pp.1269-1284. 10.1007/s10531-016-1143-5 . hal-01444012

\author{
HAL Id: hal-01444012 \\ https://hal.science/hal-01444012
}

Submitted on 21 Feb 2022

HAL is a multi-disciplinary open access archive for the deposit and dissemination of scientific research documents, whether they are published or not. The documents may come from teaching and research institutions in France or abroad, or from public or private research centers.
L'archive ouverte pluridisciplinaire HAL, est destinée au dépôt et à la diffusion de documents scientifiques de niveau recherche, publiés ou non, émanant des établissements d'enseignement et de recherche français ou étrangers, des laboratoires publics ou privés. 


\title{
Biodiversity knowledge synthesis at the European scale: actors and steps
}

\author{
Barbara Livoreil ${ }^{1,2} \cdot$ Ilse Geijzendorffer ${ }^{3} \cdot$ Andrew S. Pullin ${ }^{2}$. \\ Stefan Schindler ${ }^{4,5} \cdot$ Marie Vandewalle ${ }^{6}$ Carsten Nesshöver ${ }^{6}$
}

Received: 12 October 2015/Revised: 24 January 2016/Accepted: 28 January 2016/ Published online: 30 May 2016

\begin{abstract}
To respond to the need for a strengthened biodiversity science-policy-society interface at the European level, this paper presents the relevant actors and steps of a knowledge synthesis process relying on a Network of Knowledge. This process aims to maximize active involvement and contribution (including holders of traditional and local knowledge), transparency, credibility, relevance and legitimacy (among other values defined during several workshops held). The presented process allows for the implementation of several synthesis methodologies, depending on the availability of resources, quantity and quality of knowledge and decided according to the expectations of the requesters and users. We put this approach in parallel with other knowledge-based recommendations and negotiation processes such as CBD and IPBES and highlight the need to encompass the diversity of approaches, values, and challenges at the European scale, while the process simultaneously has to be highly flexible, yet simple and robust. Although
\end{abstract}

Communicated by David Hawksworth.

This is part of the special issue on Networking Biodiversity Knowledge.

$\triangle$ Barbara Livorei

barbara.livoreil@fondationbiodiversite.fr

1 Fondation pour la Recherche sur la Biodiversité (FRB), 195 rue Saint Jacques, 75005 Paris, France

2 Centre for Evidence-Based Conservation (CEBC), Bangor University, Bangor, Gwynedd LL57 2UW, UK

3 Institut Méditerranéen de Biodiversité et d'Ecologie marine et continentale (IMBE), Aix Marseille Université, CNRS, IRD, Avignon Université, Technopôle Arbois-Méditerranée, Bât. Villemin - BP 80, 13545 Aix-en-Provence cedex 04, France

4 Department of Biodiversity and Nature Conservation, Environment Agency Austria, Spittelauer Lände 5, 1090 Vienna, Austria

5 Division of Conservation Biology, Vegetation and Landscape Ecology, University of Vienna, Rennweg 14, 1030 Vienna, Austria

6 Department of Conservation Biology, UFZ -Helmholtz-Centre for Environmental Research, Permoserstrasse 15, 04318 Leipzig, Germany 
the presented process still holds several challenges, it offers a step forward in the development and reflections on science-policy-society interfaces, based on consultations with a significant number of the actors from the European policy-science community.

Keywords Science policy interface - Knowledge synthesis - Decision making · Evidence Stakeholder involvement

\section{Introduction}

\section{The context}

Answering the need of decision makers for answers to biodiversity questions can be organised in different ways (Dicks et al. 2014). For instance, the CBD and its Subsidiary Body on Scientific, Technical and Technological Advice (SBSTTA) rely on expert consultation and expert workshops to provide condensed knowledge to Member States which can then be used to develop recommendations and suggest actions at the Conference of the Parties (COP CBD) convened every 2 years. The CBD addresses numerous topics related to biodiversity, yet it does not answer specific requests, and considers only the global scale, meaning that each Member State is thereafter in charge of implementing actions to meet the CBD targets and reports on it. The establishment of the Intergovernmental Platform on Biodiversity \& Ecosystem Services (IPBES) in 2012 (www.ipbes.net, Opgenoorth and Faith 2014; Diaz et al. 2015) created a mandate and a driver at the global and regional level to better organise knowledge flows to address key issues related to biodiversity and ecosystem services. IPBES also contributes to capacity building science-society interfaces and to integrate different data types in its assessments (UNEP 2013, Decision IPBES-2/5). IPBES-assessments of knowledge are conducted by nominated scientific experts in working groups or Taskforces. Resulting expert-based reports are reviewed by peer experts, governments and stakeholders (UNEP 2013, Decision IPBES-2/3, 3.1 (1) \& (n)). These IPBES assessments belong to a multi-annual work programme approved by the Plenary and this programme addresses a precise set of questions of global importance, within a delineated time frame of 5 years, currently 2014-2018. It also includes work implemented as Regional Assessments, such as for the region of Europe and Central Asia. There have also been initiative regarding synthesis needs that have come from the research community. For instance, several centres, such as sDiv, CESAB, ACEAS and others (see for instance Lynch et al. 2015; Specht et al. 2015) have been created to catalyse transdisciplinary synthesis in ecosystem science and management by relying on expert groups and diverse knowledge synthesis methods. Some of these centres form the International Synthesis Consortium (http://synthesis-consortium.org) acting as a network and aiming at facilitating synthesis of diverse and disparate existing datasets.

The development of syntheses and networks to provide knowledge to decision-makers has also been of concern at the European level. Targeted European projects such as BiodiversityKnowledge (KNEU, www.biodiversityknowledge.eu/KNEU, see also Nesshöver et al. 2016, this issue) and the European Biodiversity Observation Network (EU BON, Hoffmann et al. 2014, http://eubon.eu/) aim to provide the knowledge base to inform European biodiversity questions, as well as to provide European contributions to larger scale platforms such as IPBES and the CBD. 


\section{The challenge}

A diversity of formal and informal knowledge and data can be used by different national and international bodies to inform on the status and trends of biodiversity and ecosystems as well as their functioning and the benefits they provide to mankind (ecosystem services). Despite the expressed need for good quality information on biodiversity trends (Tittensor et al. 2014), current data and knowledge flows between producers and users are still impaired by considerable barriers. These barriers include for instance silo thinking (Kay and Regier 2000; Fairbrass and Jordan 2004; Young et al. 2014), mismatches between scientific aims and societal or policy needs (Amano and Sutherland 2013; Neßhöver et al. 2014), the complexity of cause-effect relationships in natural and human-influenced systems and the diversity of values and objectives (Sarkki et al. 2013; Young et al. 2013; 2014). Thus, decision-makers often face contradictory or incomplete knowledge, unexplained variability of scientific results, discrepancies with traditional empirical knowledge, and little predictability of the consequences of their decisions. This can lead to using knowledge or handpicked expert opinions selectively (Pielke 2007; Dicks et al. 2014). The concept of evidence-based decision-making highlights the need to improve the use of evidence in informing the choice among different options and/or actions. Methods such as systematic reviews or systematic maps aim at examining the largest available pieces of evidence in an objective way, relying on explicit criteria and steps. Yet, as data or strong evidence is not always available or applicable, complementary approaches are required, e.g. adaptive management approaches, relying on an iterative "learning by doing" process, bringing in empirical evidence once the actions put in place. The portfolio of methodologies that can be used for knowledge synthesis is even wider, including different forms of balanced expert consultation (Sutherland and Burgman 2015). Enlarged consultations of knowledge holders and stakeholders, like those planned by IPBES, aim at encompassing various types of knowledge and know-hows, including traditional and technical aspects. This is considered to be of key importance for evaluating risks and feasibility (Tengö et al. 2014; Sutherland et al. 2013). As a result, the choice of methodology mainly depends on the question at stake, the knowledge needed, its availability, sources and the level of risk manageable in the policy context (see Pullin et al. 2016, this issue).

Concerns regarding biased representativeness, influence of charismatic persons, or conflicts of interest of experts and stakeholders often rise when simple consultations are conducted (Sutherland and Burgman 2015). Credible, legitimate and relevant knowledge is often resulting from a trade-off between the holders of different views (Cash et al. 2003, Sarkki et al. 2013). This trade-off may not simply be the consequence of a linear transfer of scientific knowledge to decision-makers. It may rather result from establishing a "conversation" process at various times before the decision is made, in order to facilitate effective knowledge exchange and co-construction of synthesis (Young et al. 2014).

The Network of Knowledge developed by BiodiversityKnowledge aims at maximizing involvement and contribution (including traditional and local knowledge), transparency, credibility, relevance and legitimacy, among other values defined during various workshops held at the European scale. It aims to respond to the demands from the European community of knowledge users to facilitate knowledge exchange among themselves as well as with all knowledge users. There have been several initiatives in Europe to link existing data sources and networks for better knowledge generation. On the policy side, the Biodiversity Information System Europe (BISE, http://biodiversity.europa.eu/) brings together major data and knowledge sources. On scientific side and besides EU BON similar initiatives exist in the 
forestry sector (Schuck et al. 2007), on freshwater biodiversity (BioFresh project www. freshwaterbiodiversity.eu), marine biodiversity (www.marineboard.eu) or citizen science (ecsa.biodiv.naturkundmuseum-berlin.de) among others. But the consortia linking experts and knowledge networks in most European projects are only temporary so far.

In this paper we present the work developed by BiodiversityKnowledge in order to provide a more stable and visible process to answer requests at the trans-national and European scale based on networks of existing structures and knowledge holders. The ability to connect such networks and actors with each other to contribute to answering requests is addressed by the network function of the Network of Knowledge (hereafter referred to as Network function). The Knowledge Synthesis function is referred to as the "Answering-Decision-Need function" in the KNEU White paper and related materials (BiodiversityKnowledge 2014). It aims to provide relevant knowledge synthesis on a request driven basis with tested methods and protocols at a European level, using the Network of Knowledge of diverse actors as the main source for contributions and building on their strengths (Nesshöver et al. 2016, this issue; BiodiversityKnowledge 2014, p.16). Here we present a model of this function that can be implemented to meet the challenges of maximal inclusiveness of knowledge holders and of an improved exchange of robust and relevant knowledge between a large community of holders and users.

\section{Materials and methods}

To gather ideas and priorities and to ensure support from the community in which biodiversity questions are posed and biodiversity knowledge is provided, a process was developed over a series of iterative workshops and additional individual interviews, ensuring the contribution of approximately 300 individuals [approximate composition $10 \%$ policy makers including DG ENV and DG RTD, $15 \%$ practitioners and about $75 \%$ scientists (BiodiversityKnowledge 2014)].

In the first regional workshop in Budapest representing countries from east Europe, project members and workshop participants identified which notions should be considered as important guidance for the development and implementation of the functions of the Network of Knowledge. Subsequent regional workshops representing respectively countries from North and South Europe (Copenhagen, Aix-en-provence) refined these notions. These three workshops convened together participants from as many European countries as possible to ensure representativeness of many visions and concerns. The call for participation was launched by local organizers towards policy-makers, scientists and practitioners. No selection of participants was undertaken. The notions of the CRELE framework (credibility, relevance and legitimacy) (Cash et al. 2003; Sarkki et al. 2013) were considered very important and used as a starting point to identify complementary notions of importance in order to ensure acceptance and ownership of the decision-making process. Complementary notions were also highlighted, such as openness, transparency, explicitness of uncertainties and limitations, self-organisation, inclusiveness (to all types of knowledge and representativeness of stakeholders) and quality assurance. From here on these notions will be referred to as the Network of Knowledge's core values (Tremblay et al. 2016, this issue). Beck et al. (2014) mentioned for IPBES the importance of delegation, accountability, representation into the analysis and negotiation of expert decisionmaking. Critics that tried to discredit the Intergovernmental Panel on Climate Change 
(IPCC) and its reports demonstrate the importance of identifying and using these core values.

Based on this framing, and by examining existing processes of knowledge assessment (e.g., those of IPBES and the Millennium Ecosystem Assessment, the systematic review approach of the Cochrane collaboration, and the Collaboration for Environmental Evidence), a first model was developed to capture the complete knowledge synthesis process in a flexible way. This model was discussed at the regional workshops mentioned previously and at various conferences and revised according to comments and suggestions. Then it was used to conduct three trial assessments (Schindler et al. 2016, this issue) and the whole process was evaluated all along its development (Carmen et al. 2016, this issue). The model is referred to as the "prototype" in the White paper and related materials (BiodiversityKnowledge 2014). Based on these experiences the model was then finalised and details to provide a more thorough linkage of the process to governance, expert availability, and funding were identified (Nesshöver et al. 2016; Görg et al. 2016, both this issue).

\section{Results}

\section{Actors and steps: description of the process}

The model is presented below as a project management process where various actors are involved in different stages which are conducted in a precise order. Compared to a traditional management plan, this model aims to be more generic and more flexible. It allows any type of request to activate the process, it ensures the opportunity to use the most appropriate methodology to answer the request, and it also relies very strongly on the Network function of the Network of Knowledge to contribute to the answer by providing knowledge and experts and critical review at different stages.

\section{The actors}

The Knowledge Synthesis function recognises four different roles for actors involved in the process: the requesters, the knowledge coordination body members, the working group members and the evaluators.

- Requesters (REQ) are those who have a need for knowledge synthesis and make a request to a regional Network of Knowledge. They can potentially come from or be composed of any stakeholder interested in benefiting from updated knowledge on biodiversity and ecosystem services for their decision-making or negotiation processes. This includes scientists, private businesses, governments, environmental managers, practitioners and other institutions and entities including platforms such as IPBES. Generally, to ensure ownership, transparency, and neutrality of the process, it should be possible for any group of knowledge users to submit a knowledge request as long as it is relevant at the European scale. However, the main requester is expected to be the European Commission.

- The Knowledge Coordinating Body (KCB) interacts directly with the requester and with the working groups and is also in charge of the management of the Network of Knowledge. The KCB is composed of a mix of experts, persons with technical skills, and project managers, playing the role of honest knowledge brokers (in the sense of 
Pielke 2007) as well as facilitators between the working groups, the requesters and the remaining community of peer experts and stakeholders. Experts involved in the KCB will not be involved in a working group (potential conflict of interest during the call for tender), but they could act as evaluators (see below). The KCB has the skills and tools to present the different methodologies suitable to the requester and to conduct a scoping exercise to preliminary estimate the quantity and type of knowledge available for the request. The scope and feasibility of the project is thus evaluated and the request is refined to match the availability of resources and the expectations of the requester regarding outputs, confidence levels, and applicability. The requester should always have some opportunity to communicate their expectations for methods (e.g. they may place constraints on time and rigour).

- The Working Groups (WGs) are in charge of developing in detail the protocol of the project, conducting the knowledge syntheses, writing the deliverables, responding to peer-review comments and amending the deliverables accordingly. To ensure independence of work and conclusions they do not interact directly with the requester during the conduct of the synthesis. WGs are composed of scientists and any relevant experts. WG are appointed by the KCB following a call for tender based on the Document of Work (see below).

- The Evaluators (EVA) are a group of peer-reviewers and act as a quality control body during the knowledge syntheses. They read and comment on protocols, preliminary drafts or reports and could also be consulted in case the project needs to be re-shaped during its conduct because of the quality or quantity of knowledge available. Finally, they validate the report for compliance with standards, objectivity and clarity for the requester before it is edited. The EVAs are experts of different kinds: scientific or technical experts who as a group have particularly solid reputation and expertise on the topic addressed by the WG and the applied methodology for the synthesis, stakeholders envisioned to be possible users of the conclusions of the synthesis, as well as requester's affiliates to facilitate ownership and understanding of the methodology, conclusions and limitations of the synthesis. Transparency and traceability of comments and answers to comments are ensured by the KCB's coordination and archiving role.

These basic roles ensure a structure to the process, but for the process outputs to be effective at the science-policy interface, timely and highly skilled communication is required. Ttranslators and disseminators can ensure requesters and other stakeholders understand and take ownership of the results, and can support the dissemination and use of these results. These roles can be taken up by various actors in the process, including some members of the working group and specialized translators (Holmes and Clark 2008; Bednarek et al. 2015).

Defining the actors and roles serves to clarify the involvement of possible contributors. Rules to select and assign contributors to the different roles need to ensure credibility, relevance and transparency and should be developed as a part of the implementation of this process with the Network of Knowledge. An incentive to participate in a Network of Knowledge is the potential to reach a broad audience, collating as many contributions as possible and facilitating the accessibility of results for all interested users. Such kind of actors and roles can be observed in various already existing science-policy bodies like IPCC and IPBES. 


\section{The steps}

The model can be described in three stages: preparing, conducting and finalizing (Fig. 1). Each stage is composed of multiple tasks that form a linear process with feedback loops and iterations from start to finish (see Schindler et al. this issue for practical examples). This general setting of preparing, conducting and finalising is common in many knowledge synthesis processes, such as IPCC, IPBES, the Millennium Ecosystem Assessment or Evidence synthesis (Cochrane Collaboration, Collaboration for Environmental Evidence, Conservation evidence).

Preparation stage The $\mathrm{KCB}$ receives a request (from a requester) in a generic format, with a certain budget estimation and a specified timeframe (Pullin et al. 2016, this issuefor a typology of requests). Transparency on the requests and their selection process is ensured by an open access to this stage, for instance by posting the request on a dedicated open-access website (See Tremblay et al. 2016, this issue). Transparency of this stage additionally offers opportunities for different stakeholders to identify each other in order to jointly address concerns, to share effort and to combine budgets for conducting requests.

Scoping and dialogue with the requester takes place to refine the initial question (Pullin et al. 2009; Schindler et al. 2016, this issue). Scoping includes a scan of existing knowledge (from literature and other sources) on the topic of the request, especially existing reviews, to get a first estimation of available knowledge and evidence and to ensure feasibility of the work. Depending on constraints such as deadlines, funding, availability and type of knowledge, this allows to propose various methodologies such as expert consultations, systematic reviews, causal chain analysis, participatory scenario building, focus groups, discourse field analysis, participatory knowledge production, multi-criteria analysis, joint fact finding among others (Pullin et al. 2016, this issue). This also permits clarification of the objectives of the requester(s) and aims at minimising unrealistic expectations. This information serves to develop a realistic planning and resource allocation to the request, and also contains the likely types of outputs, timeline, work plan and expected risks and uncertainties. This composes the "Document of Work" (DoW) which must be approved by the requester, and serves as a basis to launch the call for tender in order to select the members of the WG.

The conducting stage The WG formed for this specific request is now using the DoW to finalise its own work plan. The gathering of knowledge from the Network of Knowledge and databases is further specified by the WG in terms of details about the methodology, quantity and quality of available knowledge, sources of knowledge, and criteria for selection or appraisal (estimating confidence/uncertainty). The choice of the methodology for synthesis is then confirmed or discussed again if needed. This results in a "protocol document" with a detailed method section explaining the scope and origin of knowledge used to answer the request, the type of analysis foreseen, and the detailed planning and resources. This "protocol document" should be peer-reviewed and circulated via an open consultation process in order to obtain agreement from the community of the Network of Knowledge. Comments received are archived and remain open-access, and may result in amendments of the protocol. As for IPCC, all comments are answered and this is made available to the Network of Knowledge. This approach simultaneously serves as a means to communicate about the project, offers the possibility to involve additional knowledge holders in the process, and helps to prevent flaws and to anticipate criticism as much as possible, before the actual work starts. The protocol aims to give all the methodological details so that the synthesis could be easily upgraded when needed in the future. After this 


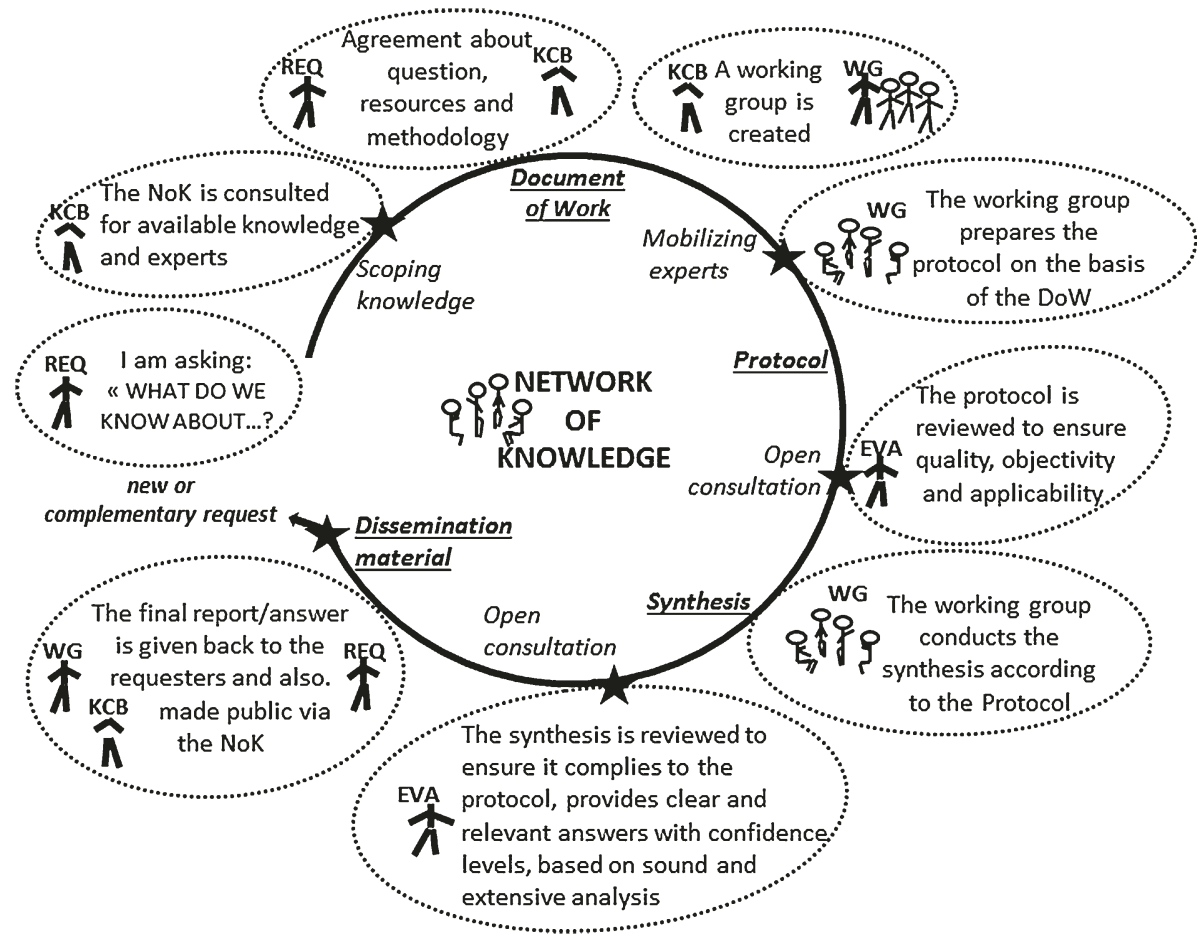

Fig. 1 Actors and steps of the process of conducting a knowledge synthesis as an answer to a European request. Stars indicate opportunities for exchange with the network of knowledge about the knowledge available, expectations, interpretation and outputs

consultative process has ended the final protocol is made open-access. The WG can start the work according to this approved protocol and produce results that will be first presented as a "draft-report".

The final stage In the finalisation stage, the draft report is subjected to peer-review and open-consultation as for the protocol. After the last adaptations, the amended document constitutes the final report in answer to the initial request. It contains recommendations for policy and management, the limitations ${ }^{1}$ observed in answering the request, as well as recommendations for further research, thus linking with the Research Strategy function of the Network of Knowledge (Nesshöver et al. 2016, this issue). The final report is delivered to the requester and results are communicated as policy briefs or via any other relevant media to interested audiences. Archiving of all steps of the work conducted ensures opportunities for efficient upgrading as well as consultation of results by any knowledge user.

As outlined in many analyses on the success or uptake of scientific input into policy processes, this final step of turning outputs of science-policy interfaces into outcomes or actions is often the most difficult one. It is subject to many external constraints, e.g. timing,

\footnotetext{
${ }^{1}$ During the conduct of the synthesis the WG may witness limitations, such as access to knowledge or data, or bias in geographical representativeness of results. These are described in a special section in the final report as "Limitations of the answer to the request" to prevent misuse or inappropriate generalisation of the answer.
} 
scope of the results in relation to the actual needs for knowledge and other sources of knowledge and interests on the according policy subject (for detailed examples, see Wilson 2009; Schindler et al. 2011; Pielke 2014). Some of these constraints are taken into account by the integrative and transdisciplinary nature of the approach presented here, because requesters and other stakeholders have the possibility to get involved in major steps of the knowledge synthesis from the start, thus enhancing ownership of the results and their potential uptake.

\section{Discussion}

\section{The network of knowledge}

The Network of Knowledge developed by BiodiversityKnowledge aims to answer requests of various types, within different timeframes, based on different knowledge and tailored to decision-makers to help them take rapid ownership of results and associated confidence (see Schindler et al. 2016, this issue for practical examples). Such a demanding objective requires $1 /$ to rely on a common and standardised process to facilitate the work of various working groups, 2/to answer in a structured and explicit way to the needs expressed by decision-makers while at the same time 3/to make sure that its chosen approach aligns with internationally accepted standards and meet criteria on rigor, credibility and transparency of the process. It also requires a high level of flexibility at various levels: e.g. application of several methodologies should be possible depending on the type of request and resources available (Pullin et al. 2016, this issue); consultation of relevant knowledge holders of the Network needs to be adjusted to the topic of the request, the work needed to answer the request may need adjustment depending on the goals, values and expectations of different groups of stakeholders.

It is generally assumed that relevant and robust knowledge can lower the risk in decision-making by informing about possible outcomes of actions, confidence, reasons for variability and sometimes even feasibility and acceptability as for instance in some systematic reviews in social sciences or in medicine (Van der Sluijs et al. 2010; Dicks et al. 2014). Given the large quantity and diversity of knowledge on many topics, this often requires some sort of synthesis in order to be transferred into a format suitable for decision making. In the biodiversity context at the international level, CBD and IPBES rely on syntheses of existing knowledge to inform decision makers (CBD technical reports, IPBES assessments). These syntheses require the gathering of data and knowledge from various locations, sources and knowledge holders. Controversies may arise when the studies used for the synthesis reach different conclusions, when vote-counting is given priority to a critical examination of the results, or when some studies are omitted for unclear reasons. Those whose voice has not been heard or taken into account in a transparent way can oppose the conclusions or withdraw their engagement and knowledge from the process. Variability in results and research designs is common in environmental sciences. The conclusions of the synthesis can highlight the heterogeneity of the underlying study results and designs, and give a level of confidence based on explicit criteria, in order to help the decision-maker assess the risk taken in implementing a related action or policy. Although such variability inhibits simple consensus based decision making, it may be crucial for the interpretation of complex issues such as biodiversity and is crucial for showcasing potential options for action (Hulme 2010). Concerning the inclusiveness of the results 
included in the synthesis, the Networking function of the Network of Knowledge is developed to maximize the participation of all knowledge holders at the European scale, yet being aware that participation in such processes is subject to many factors, including interest on the topic, access to information, and time and resource constraints.

The process of the knowledge synthesis presented here aims to provide guidance to succeed at synthesizing varied qualities and quantities of knowledge whilst still ensuring rigor, credibility and transparency. It benefited from exchanges with existing sciencesociety networks of knowledge in other disciplines like medicine (the Cochrane collaboration, www.cochrane.org) or social sciences (Campbell collaboration www. campbellcollaboration.org) as well as environment (Collaboration for Environmental Evidence, www.environmentalevidence.org). The main assets that need to be highlighted when implementing the process are:

- Participation transparent opportunities for participation can occur at different stages, as direct actors during the open consultation process, or by providing any type of knowledge to the KCB that can transmit it to the relevant WGs.

- Relevance by the dialogue with the requester at very early stages of the synthesis to define SMART goals and clarify expectations, resources, and finalise a request that is explicit for the greatest number, who can access it openly. Relevance is also ensured by regular open consultation of the Network of Knowledge, which allows to assess feasibility, possible criticisms and limitations of the answer, and acceptability of outputs by knowledge users and stakeholders.

- Flexibility stakeholders, requesters, methodologies for synthesis, types and quantity of knowledge, and statistical analyses can vary while still agreeing with the process, the backbone of which relies on a common methodology where confidence is evaluated based on transparent criteria and schools of thoughts are taken into account in trying to report values or theories that can affect the conclusions of the synthesis.

- Transparency which is looked for at all stages, especially in the protocol and final report, explaining scope, criteria for decision during the conduct of the synthesis and facilitating replicability and upgradability. Also, when the protocol is used in an open consultation process addressing the Network of Knowledge, it ensures that some criticisms or flaws can be tackled prior to the conduct of the work, relevance of the expected outcomes are discussed, knowledge providers are aware of an opportunity for contribution, and possible limitations in scope and outcomes are foreseen early in the process.

- Rigour is ensured by at least one peer-review process (report) and possibly another peer-review at an early stage (protocol). Rigour is linked to transparency but also to the methodology used by the WG (Pullin et al. 2016, this issue) as well as the rights and rules of the governance of the Network of Knowledge (Görg et al. 2016, this issue).

- Simplicity the steps of the structured Knowledge Synthesis function are those of a traditional project management plan. They provide guidance to any group conducting a work to answer a request. This simplicity also makes it very flexible to many types of requests and answers, without impairing on the other assets mentioned above.

The CRELE elements are present as core values for the Network of Knowledge and its process steps. Relevance has been already mentioned above. Credibility is linked to the transparency, rigor and simplicity of the process and the availability of the KCB to provide information, opportunities to contribute and explanations on the on-going synthesis. Legitimacy comes from inclusiveness in participation and transparency, as the Knowledge Synthesis function does not replace any of the knowledge providers but rather facilitates an 
inclusive contribution to the answer. Legitimacy, as well as credibility, build up progressively from the acknowledgment of the quality of the work provided.

\section{Comparison with other assessment procedures}

Compared to the processes established in the CBD and IPBES, the structure of the Knowledge synthesis function presents some obvious similarities (Figs. 2, 3). The main similarity is the general way assessments and syntheses can be conducted in answer to requests via a preparation, conducting and finalising phase. Yet, the Knowledge Synthesis function also presents several innovations linked to a lighter and more flexible structure. The KCB is the management unit of the whole process rather than having different structures (i.e. Plenary, Bureau, Secretariat, Multidisciplinary Expert Panel) as in IPBES. The WGs are open and not limited in size, and the community of the Network of Knowledge is consulted regularly before and during the conduct of the knowledge syntheses. This consultative process is potentially larger than that observed for CBD (esp. SBSTTA) as well as IPBES, where registered stakeholders can comment on documents, contribute to the elaboration of the work programme or identification of emerging issues, and can observe Plenaries. The inclusion of technical and local knowledge is facilitated by regular exchanges with the Network of Knowledge and the European scale and offers the

BiodiversityKnowledge

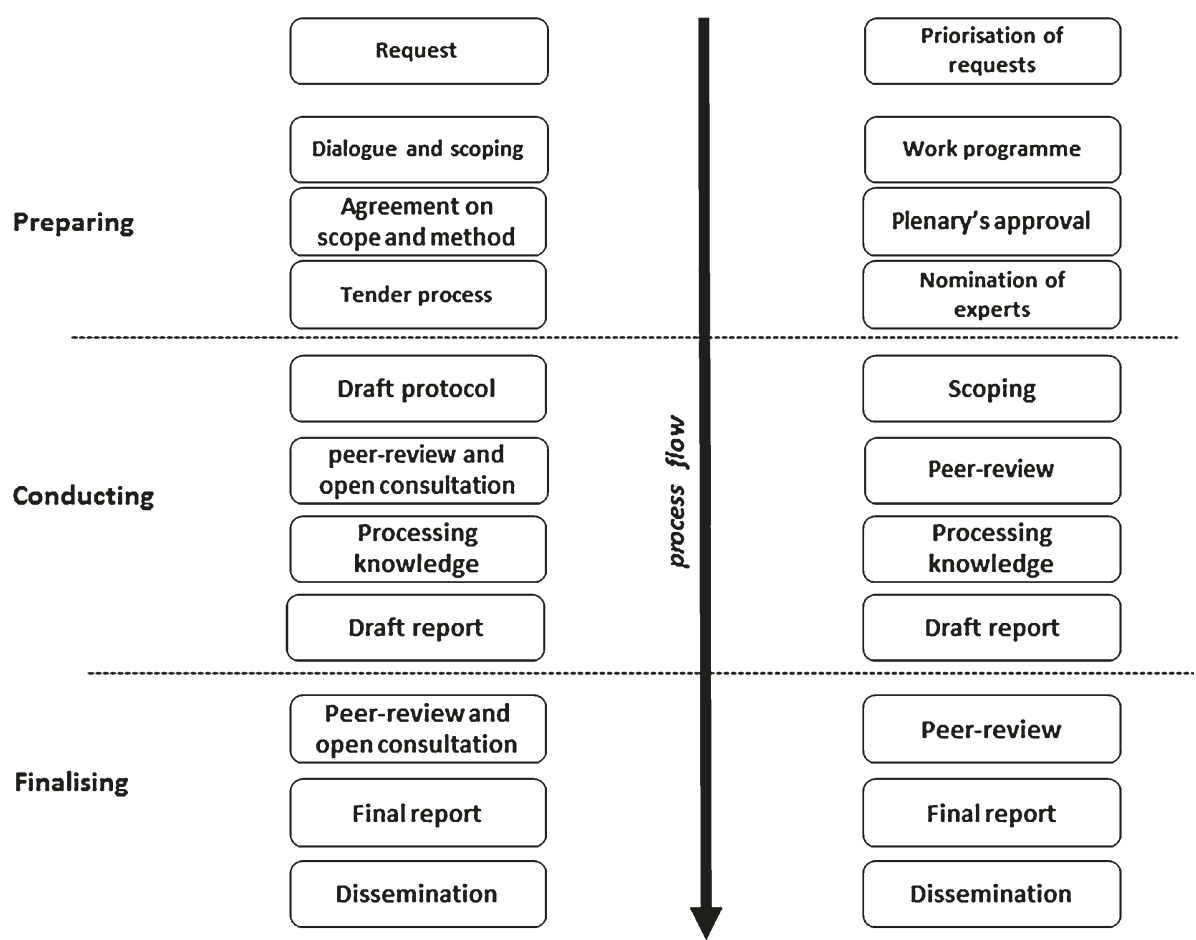

Fig. 2 Comparison of the steps of a synthesis between the Network of Knowledge and the Intergovernmental Platform on Biodiversity \& Ecosystem Services (IPBES) 


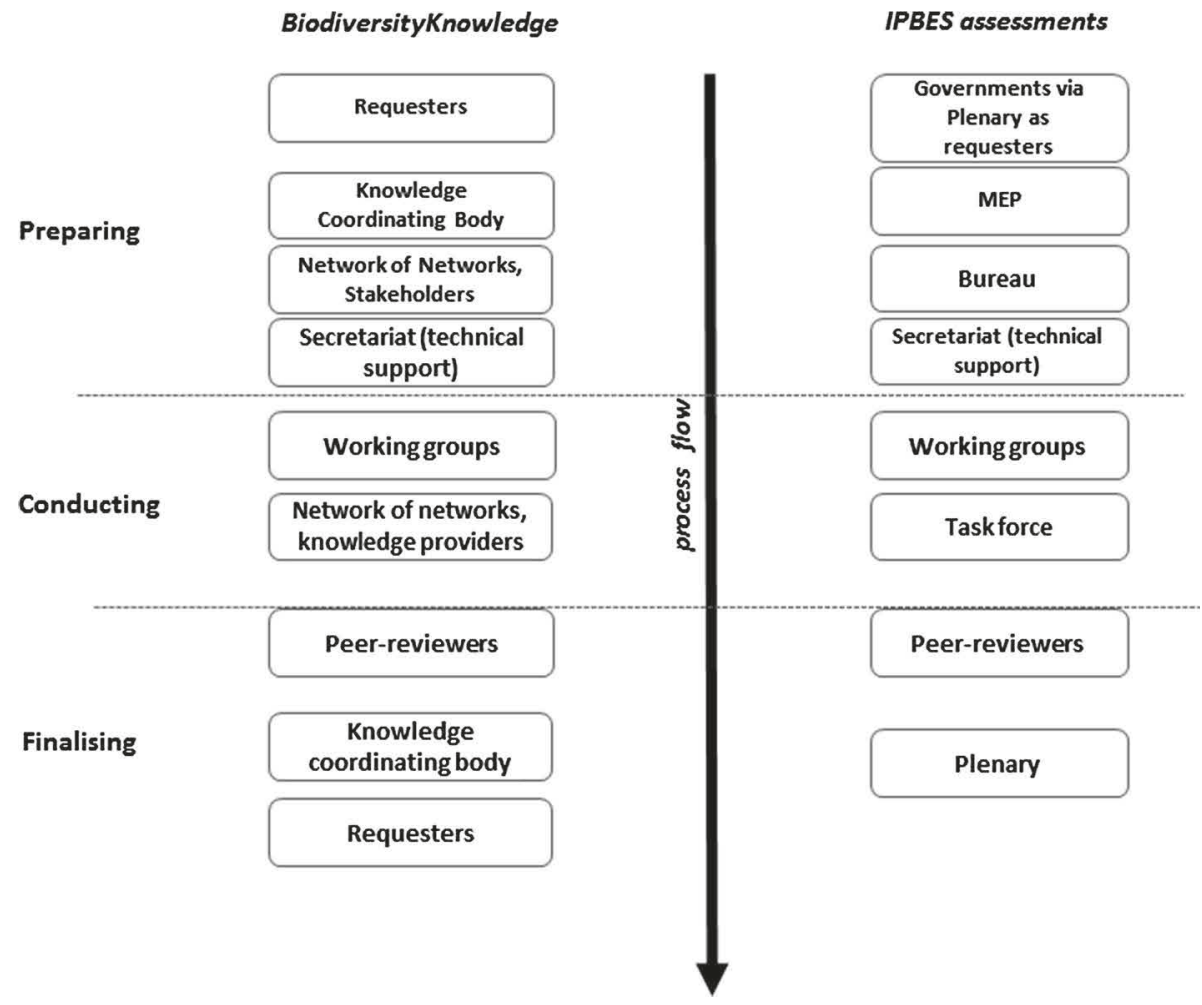

Fig. 3 Comparison of the actors involved in the Network of Knowledge and those contributing to the Intergovernmental Platform on Biodiversity \& Ecosystem Services (IPBES)

potential to scale down to a formulation of problems that indigenous experts can more easily relate and contribute to. Yet, major problems to actually access and integrate such knowledge will remain (Hernández-Morcillo et al. 2013; Tengö et al. 2014) and will need additional efforts in the context of a Network of Knowledge that in first place accessed scientific knowledge holders.

Existing platforms and bodies that currently have a mandate to provide a Knowledge Synthesis function at European level for biodiversity knowledge, include the European Environmental Agency (or other EU agencies) and the Joint Research Centre. ${ }^{2}$ However, they only provide information to answer knowledge requests shared at a European level or posed by the European Parliament or Commission. In addition, as knowledge is synthesized based on published studies and datasets by either employees of the institutes or by invitations of individual expert(s), core values of transparency and inclusiveness are not always fulfilled. The results of these studies are widely distributed and openly available, but validation or recommendations of revisions can only be provided after publications of the results.

\footnotetext{
${ }^{2}$ The interpretation of the mission of JRC regarding its function for the European Commission is likely to change in autumn 2015, due a change in mandate and a reorganisation. So the presented description about the JRC could be considered of limited validity in the near future.
} 
The implementation of a Network of Knowledge and its knowledge synthesizing function, would likely be of great interest to both the EEA and the JRC. For the EEA for example, it would be possible to link updates on biodiversity indicators in a timely manner directly with holders of evidence (e.g. monitoring data). For JRC, it would render data and knowledge accessible that could be used to fill in existing data gaps and to strengthen estimations presented by preliminary results with feedback from the community.

\section{Remaining challenges and steps forward}

The remaining challenges are numerous and we only list here those identified in previous work with groups of experts as most relevant to the process and the knowledge transfer to decision-makers (e.g. Carmen et al. 2016; Schindler et al. 2016, both this issue). A more detailed section about the challenges faced by the Network of Knowledge is presented in Nesshöver et al. (2016, this issue).

- Maintaining sufficiently engaged knowledge holders in the Network of Knowledge to contribute to syntheses but also to comment, peer review and feedback will be successful only if the exercise is rewarding (Schindler et al. 2016, this issue). The incentives could be an increased contribution to policy development from the scientific community, the opportunity to rapidly spread information about new robust results or unpublished ones (e.g. non-significant results from well-designed experimentations), the contribution to new outputs (syntheses) and outcomes (changes in policy, new knowledge-based policies), the acknowledgment of the variability of results and conclusions depending on the context (van der Sluijs et al. 2010) and a more concerted and efficient dissemination of outputs. Last but not least, the scientific outcomes, e.g. new contacts with colleagues via working groups, joint publications and new ideas for future research are relevant incentives for involved experts (Carmen et al. 2015).

- Answering criticisms (e.g. accusation of bias) about the conclusions of a synthesis will remain a challenge that will vary according to the methodology used for the synthesis. This poses the challenge of the choice of the methodology of the synthesis and the necessary trade-off between rigor and required resources allocated to the working group.

- A lot of credibility and inclusiveness of the process presented herein relies on open participation and open consultation. An underlying assumption concerns the availability and broad representation of different forms of knowledge, opinions and experts. Prior experience, especially for broad systematic reviews, or when trying to consult numerous experts suggest that there are few feedbacks and they can be biased towards those opposing the conclusions of the synthesis. In addition, voluntary contributions tend to decrease drastically with increasing time and resource demands (Schindler et al. 2016, this issue). This underlying assumption of the process and its governance will need to be addressed.

The challenges outlined above are not entirely new when the goal is to make sciencepolicy interface activities more effective (e.g., Cash et al. 2003; Hulme 2010; Sarkki et al. 2013). They match challenges identified for successful transboundary work and the way scientific and other knowledge can be assessed and used sensibly to come to better decisions (e.g, Cornell et al. 2013). A recent H2020 call has envisioned a possible future for the Network of Knowledge, namely SC5-10c-2015 "An EU support mechanism for evidencebased policy on biodiversity \& ecosystem services". The text calls for the "setting up of an innovative, self-sustainable governance mechanism (...) to enhance effective and efficient 
interactions between science, society and policy (...). This should build on existing science-policy interfaces and include all EU Member States, Associated or Accession Countries and should be open to observers". What is now needed for the Network of Knowledge, its process and all the aspects presented in various papers in this issue is to be put in practice, in an adaptive and iterative manner, in order to gain the commitment and contribution of the broad community of knowledge holders in Europe and add-up to the synthesis and assessment already conducted by IPBES and CBD, and others, to achieve better results in sustainable development, conservation of biodiversity and ecosystems and climate change adaptation.

Acknowledgments We are grateful for the contributions of our colleagues from the KNEU-consortium as well as to all policy-makers, practitioners and scientists who participated in the consultations. The work presented in this paper has been partly financed by the European Commission via its 7th framework programme. The main development of the BiodiversityKnowledge mechanism was conducted in the KNEU project (Grant No. 265299). I.R.G. was financed by the EU BON project (Grant No. 308454) and contributes to the Labex OT-Med (No. ANR-11-LABX-0061) funded by the French Government through the A*MIDEX project (No. ANR-11-IDEX-0001-02).

Compliance with ethical standards

Conflict of interest The authors declare that they have no conflict of interest.

\section{References}

Amano T, Sutherland WJ (2013) Four barriers to the global understanding of biodiversity conservation: wealth, language, geographical location and security. Proc R Soc B 280:20122649

Beck S, Borie M, Chilvers J et al (2014) Towards a reflexive turn in the governance of global environmental expertise. The cases of the IPCC and the IPBES. GAIA 23(2):80-87

Bednarek AT, Shouse B, Hudson CG, Goldburg R (2015) Science-policy intermediaries from a practitioner's perspective: the Lenfest Ocean program experience. Sci Public Policy. doi:10.1093/scipol/ scv008

BiodiversityKnowledge (2014) A recommended design for "Biodiversity Knowledge", a network of knowledge to support decision making on biodiversity and ecosystem services in Europe." White paper. UFZ, Leipzig

Carmen E, Neßhöver C, Saarikoski H, Vandewalle M, Watt A, Görg H, Young J (2015) Creating a biodiversity science community: experiences from a European network of knowledge. Environ Sci Policy 54:497-504

Carmen E, Watt A, Saarikoski H, Young J (2016) Participation and scale: focusing on learning as an outcome to improve participation at larger scales. Biodivers Conserv

Cash DW, Clark WC, Alcock F et al (2003) Knowledge systems for sustainable development. Proc Natl Acad Sci 100:8086-8091

Cornell S et al (2013) Opening up knowledge systems for better responses to global environmental change. Environ Sci Policy 28:60-70. doi:10.1016/j.envsci.2012.11.008

Diaz S et al (2015) The IPBES conceptual framework - connecting nature and people. Curr Opin Environ Sustain 14:1-16

Dicks L, Walsh JC, Sutherland WJ (2014) Organising evidence for environmental management decisions: a '4S' hierarchy. Trends Ecol Evol 29:607-613

Fairbrass J, Jordan A (2004) Multi-level governance and environmental policy. In: Bache I, Flinders MV (eds) Multi-level governance. Oxford University Press, Oxford, pp 147-164

Görg C et al (2016) The Governance of Science-Policy Interfaces - Network vs. Platform approach. Biodivers Conserv

Hernández-Morcillo M, Hoberg J, Oteros-Rozas E et al (2013) Traditional ecological knowledge in Europe: status quo and insights for the environmental policy agenda. Environ: Sci Policy Sustain Dev 56:3-17. doi:10.1080/00139157.2014.861673

Hoffmann A, Penner J, Vohland K et al (2014) Improved access to integrated biodiversity data for science, practice, and policy - the European biodiversity observation network (EU BON). Nat Conserv 6:49-65 
Holmes J, Clark R (2008) Enhancing the use of science in environmental policy-making and regulation. Environ Sci Policy 11:702-711

Hulme M (2010) Problems with making and governing global kinds of knowledge. Glob Environ Change 20:558-564

Kay J, Regier H (2000) Uncertainty, complexity, and ecological integrity: insights from an ecosystem approach. In:Crabbe P, Holland A. et al (eds) Implementing ecological integrity: restoring regional and global environmental and human health, Kluwer, NATO Science Series, Environmental Security pp 121-156

Lynch AJJ, Thackway R, Specht A et al (2015) Transdisciplinary synthesis for ecosystem science, policy and management: the Australian experience. Sci Total Environ 534:173-184. doi:10.1016/j.scitotenv. 2015.04.100

Neßhöver C, Ten Brink P, Balian E et al. (2014) Summary report and recommendations on improving the Science-policy interface for biodiversity and ecosystem services in Europe (contract: Ref No 07-0307/ 2013/661961/SER/B2). http://ec.europa.eu/environment/nature/knowledge/pdf/EU\%20Mechanism\% 20Summary\%20Report\%202015.pdf

Nesshöver C et al (2016) The network of knowledge approach - improving the science and society dialogue on biodiversity and ecosystem services in Europe. Biodivers Conserv

Opgenoorth L, Faith DP (2014) The intergovernmental science-policy platform on biodiversity and ecosystem services (IPBES), up and walking. Front Biogeogr 5:207-211

Pielke RA Jr (2007) The honest broker-making sense of science in policy and practice. Cambridge University Press, UK, p 188

Pielke RA Jr (2014) The rightful place of science: disasters and climate change. Arizona State University, Tempe, p 124

Pullin AS, Knight TM, Watkinson AR (2009) Linking reductionist science and holistic policy using systematic reviews: unpacking environmental policy questions to construct an evidence-based framework. J Appl Ecol 46:970-975

Pullin AS et al (2016) Selecting appropriate methods of knowledge synthesis to inform biodiversity policy. Biodivers Conserv

Sarkki S, Niemelä J, Tinch R, van den Hove S, Watt A, Young J (2013) Balancing credibility, relevance and legitimacy: a critical assessment of trade-offs in science-policy interfaces. Sci Public Policy 41:194-206. doi:10.1093/scipol/sct046

Schindler S, Curado N, Nikolov S, Kret E, Cárcamo B, Poirazidis K, Catsadorakis G, Wrbka T, Kati V (2011) From research to implementation: nature conservation in the Eastern Rhodopes mountains (Greece and Bulgaria), European Green Belt. J Nat Conserv 19:193-201

Schindler S et al (2016) The network biodiversity knowledge in practice: insights from three trial assessments. Biodivers Conserv

Schuck A et al (2007) Towards a European forest information system. European Forest. Institute Research Reports 20, Brill

Specht A, Gordon IJ, Groves RH, Lambers HG, Phinn SR (2015) Catalysing transdisciplinary synthesis in ecosystem science and management. Sci Total Environ 534:1-3

Sutherland WJ, Burgman MA (2015) Use experts wisely. Nature 526:317-318

Sutherland WJ, Gardner TA, Haider L, Dicks L (2013) How can local and traditional knowledge be effectively incorporated into international assessments? Oryx 48:1-2

Tengö M, Brondizio ES, Elmqvist T, Malmer P, Spierenburg M (2014) Connecting diverse knowledge systems for enhanced ecosystem governance: the multiple evidence base approach. Ambio 43:579-591. doi:10.1007/s13280-014-0501-3

Tittensor DP, Walpole M, Hill SLL et al (2014) A mid-term analysis of progress towards international biodiversity targets. Science 346:241-244

Tremblay M, Vandewalle M, Wittmer H (2016) Ethical challenges in an open system: the network of knowledge's ethical risks assessment and its ethical infrastructure. Biodivers Conserv

UNEP (2013a) Decision IPBES-2/3 - procedures for the preparation of the platform's deliverables. Secretariat of the Intergovernmental Platform on Biodiversity and Ecosystem Services, Bonn

UNEP (2013b) Decision IPBES-2/5-work programme for the period 2014-2018. Secretariat of the Intergovernmental Platform on Biodiversity and Ecosystem Services, Bonn

Van der Sluijs JP, Van Est R, Riphagen M (2010) Beyond consensus: reflections from a democratic perspective on the interaction between climate politics and science. Curr Opin Environ Sustain 2(5):409-415

Wilson DC (2009) The paradoxes of transparency: science and the ecosystem approach to fisheries management in Europe. Amsterdam University Press, Amsterdam, p 303 
Young JC, Watt AD, van den Hove S et al. (2013). Effective interfaces between science, policy and society: the SPIRAL project handbook. http://www.spiralproject.eu/content/documents

Young JC, Waylen K, Sarkki S et al (2014) Improving science-policy dialogue to meet the challenges of biodiversity conservation: having conversations rather than talking at one-another. Biodivers Conserv 23:387-404 